\title{
The role of memory representation in the vigilance decrement
}

\author{
DANIEL M. CAGGIANO and RAJA PARASURAMAN \\ Catholic University of America, Washington, D.C.
}

\begin{abstract}
Working memory load is critically important for the overall level of performance on vigilance tasks. However, its role in a key aspect of vigilance-sensitivity decrement over time-is unclear. We used a dual-task procedure in which either a spatial or a nonspatial working memory task was performed simultaneously with a spatial vigilance task for $20 \mathrm{~min}$. Sensitivity in the vigilance task declined over time when the concurrent task involved spatial working memory. In contrast, there was no sensitivity decrement with a nonspatial working memory task. The results provide the first evidence of a specific role for working memory representation in vigilance decrement. The findings are also consistent with a multiple resource theory in which separate resources for memory representation and cognitive control operations are differentially susceptible to depletion over time, depending on the demands of the task at hand.
\end{abstract}

Most people find it difficult to sustain attention on one source of information for an extended period of time. If one needs to monitor that source to detect a rare, unpredictable target among frequent nontargets - for example, an airport security screener checking baggage for a weapon - the accuracy or speed of detection typically deteriorates over time, a phenomenon known as vigilance decrement (Davies \& Parasuraman, 1982). Although William James (1890) presciently noted that "there is no such thing as voluntary attention sustained for more than a few seconds" (p. 420), vigilance decrement typically occurs after $20-30 \mathrm{~min}$ of continuous work but can occur under certain conditions in as little as $5 \mathrm{~min}$ (Nuechterlein, Parasuraman, \& Jiang, 1983).

Because a decline in hit rate for targets can result from changes in either perceptual sensitivity $\left(d^{\prime}\right)$ or the observer's decision criterion ( $\beta$; Davies \& Parasuraman, 1982 ), uncovering the key factor contributing to performance declines is important both theoretically and pragmatically. Vigilance paradigms have been used widely to assess the risk of performance deterioration in various work settings, as well as to examine and remediate attentional deficits in clinical populations.

In an earlier examination of the issue, Parasuraman (1979) showed that a sensitivity decrement occurs primarily

This research was supported by NIH Grant AG19653 from the National Institute of Aging to R.P. We thank Arthur D. Fisk, Brian H. Ross, Joel S. Warm, Pamela M. Greenwood, Shimin Fu, and an anonymous reviewer for helpful comments on earlier drafts of this article, Yang Jiang, Xiong Jiang, and the members of the Cognitive Science Laboratory cognitive neuroscience journal club for discussions on experimental design, and Melodie Harris for assistance in task programming. Correspondence concerning this article should be addressed to D. M. Caggiano, Cognitive Science Laboratory, Catholic University of America, 250 O’Boyle Hall, Washington, DC 20064 (e-mail: 81caggiano@cua.edu). when the observer has to discriminate targets from nontargets represented in working memory (WM). When stimulus events were presented rapidly in such successivediscrimination tasks, sensitivity declined markedly over time on task. In contrast, if target discrimination did not load memory, as when target and nontarget features were presented concurrently (simultaneous discrimination), sensitivity remained stable over time. Parasuraman (1979) linked the sensitivity decrement over time in successivediscrimination tasks to the working memory requirement and to the depletion of attentional resources throughout the vigil.

The view that successive-discrimination vigilance tasks are more resource demanding than simultaneous tasks because of the inherent WM load has been supported in a number of studies (see Warm \& Dember, 1998). For example, Gluckman, Dember, and Warm (1988) examined patterns of dual-task interference for different pairs of successive or simultaneous vigilance tasks. They found that the degree of dual-task decrement in sensitivity increased progressively as the number of successive tasks in each dualtask pair increased from zero, to one, to two. Nevertheless, the view that the sensitivity decrement over time occurs only in successive tasks at high event rates was not confirmed in some subsequent studies, in which the overall level of performance, but not the decrement, was affected by memory load and task demand manipulations (Warm \& Dember, 1998). Furthermore, shortly after the Parasuraman (1979) report, Nuechterlein et al. (1983) demonstrated a sensitivity decrement over time in a simultaneous-discrimination task for perceptually degraded stimuli. Parasuraman and Mouloua (1987) reported a sensitivity decrement at all three levels of target discriminability for a successive task but also found a decrement in a simultaneous task at a low level of discriminability. These and other studies led to the conclusion that although WM load is important for the 
overall level of vigilance performance, the sensitivity decrement may result from any factor (including memory load) that increases the overall demand for attentional resources (Warm \& Dember, 1998). This nonspecific resource view of vigilance decrement is consistent with the distinction between automatic and controlled processing made by Fisk and Schneider (1981), in which simultaneous tasks can be performed automatically, whereas successive tasks require continuous cognitive control, rendering them more susceptible to decrement over time.

The role of WM in vigilance decrement is thus unclear, despite evidence from two other relevant studies. Todkill and Humphreys (1994) demonstrated that short-term and long-term memory strategies can be applied to vigilance paradigms, with both yielding declines in sensitivity over time; however, the rate of performance decline over time did not differ for these two memory representation strategies. Thus, a decline in the efficiency of the cognitive control processes (e.g., Fisk \& Schneider, 1981) common to both the long-term and the short-term memory systems may have caused the vigilance decrement in both conditions. Baddeley, Cocchini, Della Sala, Logie, and Spinnler (1999) found that detection rate declined more over time for a successive than for an equally difficult simultaneous vigilance task in Alzheimer's disease (AD) patients, whereas no decrement in either task was observed in elderly controls. Baddeley et al. attributed this difference in vigilance performance to $\mathrm{WM}$ deficits in $\mathrm{AD}$, but more specifically, to deficient executive control.

Both of these studies attest to some involvement of WM processes in vigilance performance, yet the question of whether memory representation plays a role in vigilance decrement remains unanswered. To address this issue, participants performed a successive-discrimination vigilance task while concurrently carrying out one of two WM tasks. In both conditions, the vigilance task involved the same absolute spatial judgment about the distance of a flashing dot from the center of the screen. The WM task loaded either spatial or nonspatial memory. We hypothesized that if memory representation modality affects the vigilance decrement, loading the spatial buffer while participants perform a spatial vigilance task would exacerbate this decline, whereas loading a nonspatial buffer would not. Signal detection theory (Green \& Swets, 1974) was used to assess changes in the $p(a)$ measure of sensitivity over time, independent of changes in decision factors.

\section{METHOD}

\section{Participants}

Twelve young adults ( 7 females) between the ages of 18 and 30 with 20/30 vision or better participated.

\section{Stimuli and Procedure}

All the stimuli were presented on a high-resolution color monitor (refresh rate of $75 \mathrm{~Hz}$ ) via a G4 Macintosh computer. The participants performed each of two dual-task conditions combining a WM task and a vigilance task.

Working memory tasks. These taks involved a two-back memory operation (Dobbs \& Rule, 1989) on either a spatial or a nonspatial aspect of overlapping, colored wrench stimuli $\left(10.3^{\circ} \times 11.2^{\circ}\right.$ of visual angle for the set of wrenches) presented centrally (see Figure 1), similar to the stimuli developed by Kramer and Weber (1998). In the spatial condition, the participants compared the location of the open wrench head with the location at which it had appeared two trials previously. In the nonspatial condition, the participants compared the color (green or purple) of the open-headed wrench with the color of the open-headed wrench from two trials previously. The participants were required to respond to a two-back match $(50 \%$ of the trials) by pressing a response key (either "Z" or " ").

Vigilance task. One thousand nine hundred milliseconds after the onset of the wrench pair, which remained on the screen for the duration of the trial, a black oval stimulus $\left(0.6^{\circ} \times 1.0^{\circ}\right)$ flashed for $200 \mathrm{msec}$ at one of two eccentricities $\left(6.9^{\circ}\right.$ or $\left.8.6^{\circ}\right)$ to either the left or the right of the wrench pair. The participants were required to press a response key (either "Z" or "'/") whenever the oval appeared at the outer of the two eccentricities ( $20 \%$ of the trials).

Each trial was followed by a 100 -msec intertrial interval of blank white screen. The mapping of the "Z" and "/" response keys to task was counterbalanced across participants.

The participants first received training on one of the dual-task conditions (e.g., location WM plus vigilance) for 200 trials over six discontinuous blocks. They then performed trials in that condition for a continuous 20-min period consisting of three consecutive blocks, each containing 100 trials. Each block contained 20 targets for the vigilance task and $50 \mathrm{WM}$ match trials. After a brief rest break, the participants received training and performed the tasks in the other dual-task condition (e.g., color WM plus vigilance). The participants completed the NASA Task Load Index (TLX) following completion of each training and vigilance session as a subjective measure of the workload associated with each condition. The order of the dual-task conditions was counterbalanced across participants.

\section{RESULTS}

\section{Vigilance Performance}

All analyses of variance (ANOVAs) used the Greenhouse-Geisser correction for sphericity. A 2 (WM task) $\times$ 3 (block) $\times 2$ (counterbalance order) repeated measures ANOVA for the $p(a)$ sensitivity measure revealed no significant main effect of WM task, indicating that the overall level of vigilance performance did not depend on which WM task was being performed concurrently $[p(a)=$ .80 for both conditions; $F(1,11)=0.00, M S_{\mathrm{e}}=0.005$, $p=.988]$. The main effect of block was significant $\left[F(1.6,17.62)=5.54, M S_{\mathrm{e}}=0.004, p<.05\right]$, indicating that a vigilance decrement was observed. WM task and block interacted significantly $\left[F(1.62,17.86)=4.56, M S_{\mathrm{e}}=\right.$ $0.003, p<.05$ ], with sensitivity declining more rapidly under the location WM condition than under the color condition (see Figure 2A). Simple effects tests confirmed this interpretation; block did not significantly affect sensitivity in the color condition $[F(1.57,17.31)=0.81$, $\left.M S_{\mathrm{e}}=0.030, p=.435\right]$, but did in the location condition $\left[F(1.63,17.91)=11.19, M S_{\mathrm{e}}=0.003, p<.005\right]$. Furthermore, sensitivity in the first block did not differ significantly between the two conditions $[t(11)=1.53, p=$ $.153]$, indicating an initial equivalency in vigilance sensitivity. None of the comparisons involving the factor of order was significant ( $p>.14$ for all comparisons).

\section{Working Memory Performance}

A 2 (WM task) $\times 3$ (block) repeated measures ANOVA showed that sensitivity was higher in the location [mean 
Trial 1

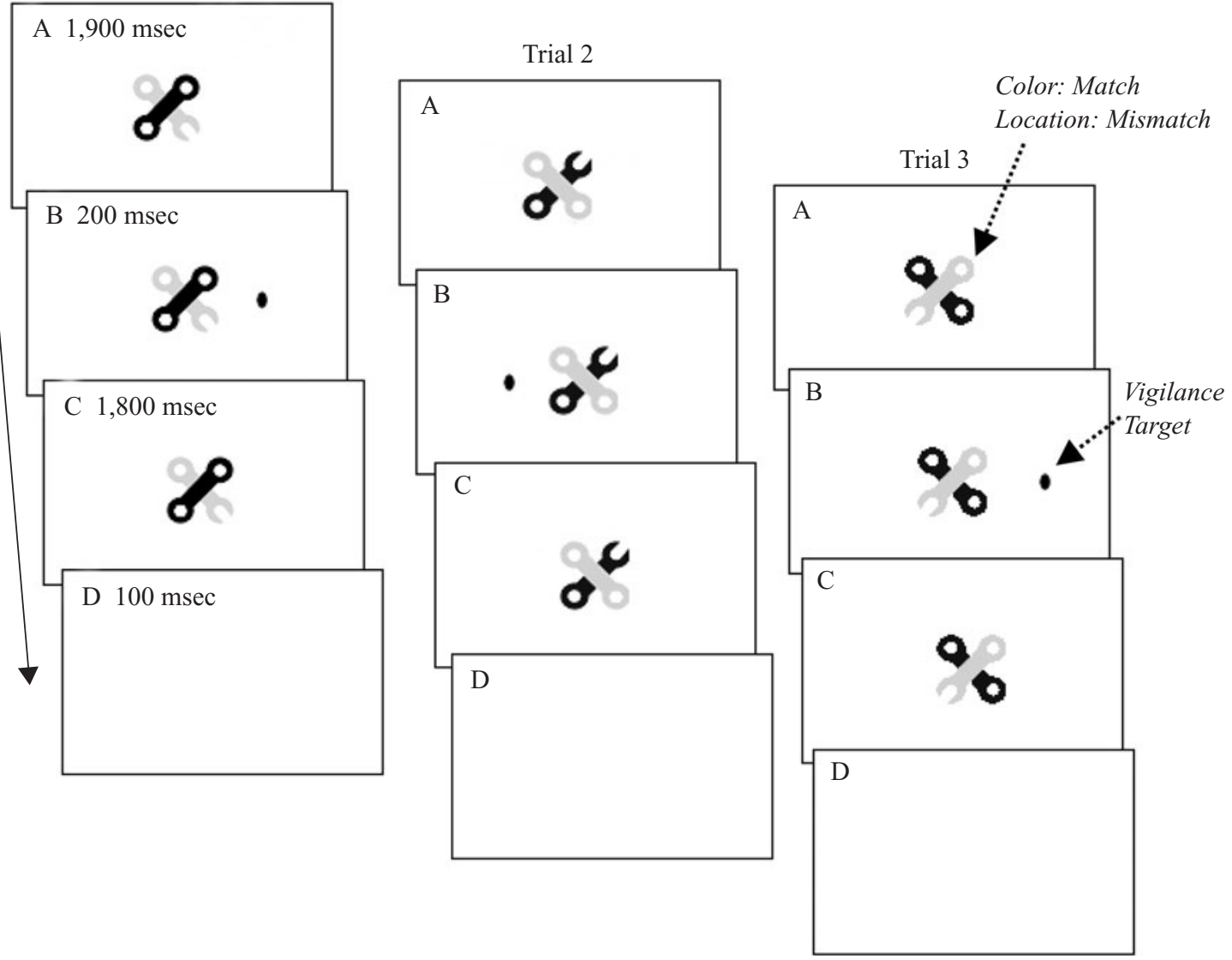

Figure 1. Stimuli for the working memory and vigilance tasks (figure not to scale). Wrench stimuli were green and purple during the actual experiment. (A) Wrench stimulus pairs appeared at the beginning of a trial and remained on the screen for the 3,900-msec trial duration. (B) A black oval flashed for 200 msec 1,900 msec after wrench pair onset, to either the right or the left of the wrench pair at one of two possible eccentricities. (C) The participants were given $1,800 \mathrm{msec}$ to respond to the ovals, followed by (D) an intertrial interval of $100 \mathrm{msec}$ prior to the onset of the next trial. The participants had to compare either the location of the open wrench head or the color of the wrench with the open head with that in the trial before the previous trial (e.g., compare panel $3 \mathrm{~A}$ with panel $1 \mathrm{~A}$ ). In the vigilance task, the participants were required to detect when the oval stimulus infrequently flashed at the outer of the two possible eccentricities.

$p(\mathrm{a})=.93]$ than in the color WM task $[$ mean $p(\mathrm{a})=.88$; $F(1,11)=9.04, M S_{\mathrm{e}}=0.045, p<.05$; see Figure 2B]. Thus, despite our effort to equate overall difficulty, the location task was somewhat easier than the color task. Neither the main effect of block $[F(1.86,20.47)=0.18$, $\left.M S_{\mathrm{e}}=0.001, p=.818\right]$ nor the task $\times$ block interaction $\left[F(1.82,19.98)=1.13, M S_{\mathrm{e}}=0.001, p=.337\right]$ was significant.

At the end of each task condition, the participants were asked to state the strategy they had used to perform the WM task. Of the 12 participants, 7 reported a verbal strategy in the color (nonspatial) task, whereas none reported a spatial strategy. In the location (spatial) task, 4 reported a spatial strategy and 1 a verbal strategy. The remainder of the participants reported no particular strategy. ${ }^{1}$

\section{Subjective Workload}

A 2 (session) $\times 2$ (condition) within-subjects ANOVA showed that perceived mental workload was higher for the 20-min vigilance sessions than for the practice sessions [mean rating on a scale of 1-100: training, 51.8; vigilance, $\left.54.7 ; F(1,11)=4.82, M S_{\mathrm{e}}=30, p=.05\right]$. Mental workload was also rated higher in the color WM condition (mean, 56.7 ) than in the location condition [mean, $59.7 ; F(1,11)=$ $\left.14.47, M S_{\mathrm{e}}=49, p<.005\right]$. The session $\times$ condition interaction was not significant $[F(1,11)=1.25, p=.287]$.

\section{DISCUSSION}

\section{Vigilance}

Target detection sensitivity in the vigilance task declined over time. This replication of vigilance decrement with a successive-discrimination task is consistent with Parasuraman (1979) and with a large body of subsequent literature in which it has been shown that vigilance tasks requiring $\mathrm{WM}$ for successful target discrimination are susceptible to diminished sensitivity over time. Furthermore, subjective mental workload increased significantly 

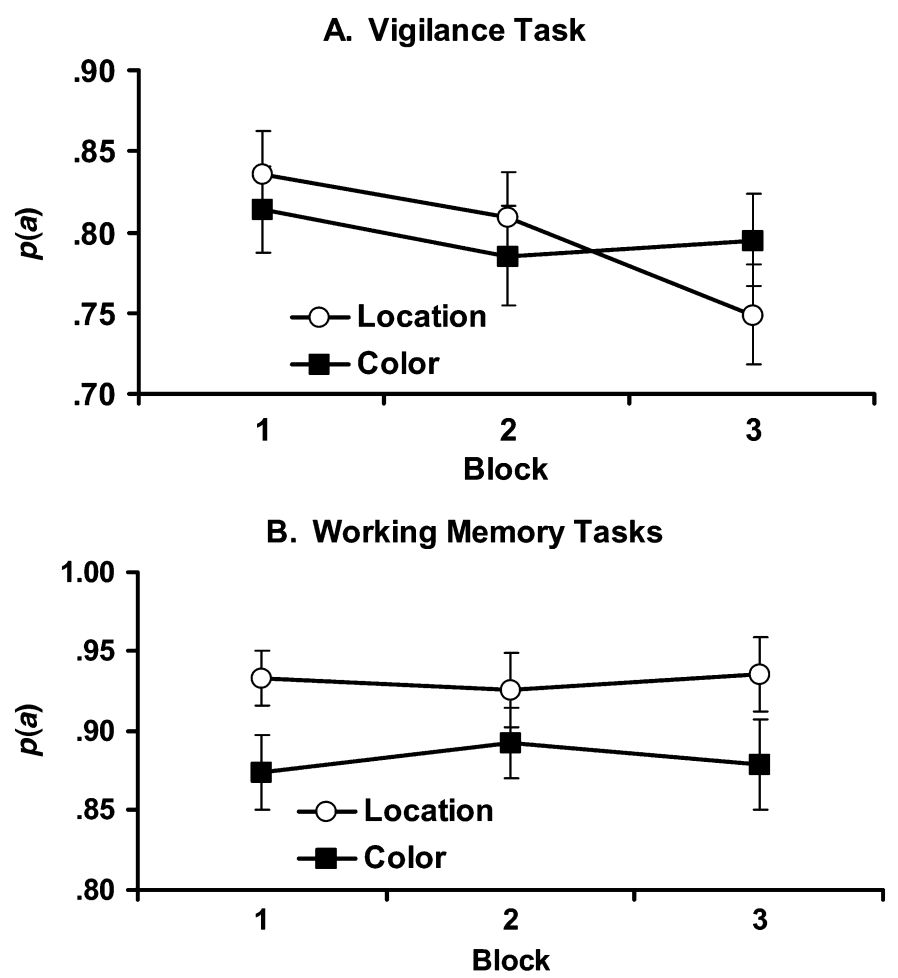

Figure 2. Sensitivity for (A) the vigilance task and (B) the two working memory tasks in each dual-task condition as a function of time on task.

from the practice to the vigilance session, again consistent with previous research (Warm \& Dember, 1998).

Although WM load interacts with other factors to influence the level of vigilance performance (Warm \& Dember, 1998), its role in vigilance decrement has not been established. Although previous researchers have suggested the importance of WM activation for vigilance decrement (Baddeley et al., 1999; Todkill \& Humphries, 1994), these studies did not dissociate the specific roles of executive control and memory storage. We designed this study to test the assertion that memory representation per se plays an important role in vigilance decrement (Parasuraman, 1979). Consistent with our hypothesis, performance on the same vigilance task declined more when it was paired with the location memory task than with the color task. This result provides strong evidence for a specific role of memory representation in vigilance decrement.

Several possible objections to this conclusion must be considered. First, despite our efforts to match the WM tasks for overall difficulty, they were not equivalent. According to both nonspecific cognitive control and central resource theories of vigilance performance (Fisk \& Schneider, 1981; Warm \& Dember, 1998), because both WM tasks required controlled processing, the more difficult of the two should have depleted attentional resources to a greater degree. Contrary to this prediction, the nonspatial WM task proved more difficult in both the objective performance (sensitivity) and the subjective (mental work- load) measures, yet no vigilance decrement in sensitivity was observed. Only in the less difficult spatial memory condition did vigilance sensitivity decline over time. A similar argument could be made about the amount of effort expended; however, a paired-samples $t$ test of the "effort" subscale of the TLX indicated no significant difference between the two vigilance sessions, making such an account unlikely. Second, any differences in initial vigilance sensitivity across the two conditions could have accounted for the difference in vigilance decrement. This possibility can also be discounted because the participants performed equally well on the vigilance task during the first block of trials in each condition. Third, the participants may have used a common strategy, such as verbal encoding, to perform both the location and the color twoback memory tasks. Although there were individual differences and many participants reported using no specific strategy, open-ended subjective reports of the participants' strategies provided explicit evidence that some participants had used the strategies we had anticipated (i.e., spatial for the location task, verbal for the color task). Furthermore, virtually no participant reported strategies other than those predicted. Finally, the spatial WM task may have elicited increasingly frequent eye movements across blocks that suppressed the participants' ability to perceive the probe. ${ }^{2}$ To test this possibility, we recorded eye movements as three additional participants performed the spatial WM condition. The number of saccades that occurred 
specifically during probe presentation did not increase across blocks, making eye movements an unlikely explanation for the sensitivity decrement in the spatial WM condition.

Our findings argue against the habituation account of sustained attention effects (Mackworth, 1969), in which repeated exposure to similar target and nontarget stimuli reduces the activation associated with each over time and, thus, the difference in activation between them, reducing discriminability. In both dual-task conditions, the participants were exposed to equal numbers of target and nontarget stimuli per block at the same presentation rate, yet detection performance declined in one condition, but not in the other. Rather, the data support a sustained demand account of vigilance decrement that incorporates multiple resource views of memory and attention (Baddeley \& Hitch, 1994; Wickens, 1984) - sustained attention taxes resources available for the memory representation store that is required. Previous research (Fisk \& Schneider, 1981; Warm \& Dember, 1998) suggests further that executive or cognitive control mechanisms may also be susceptible to resource depletion over time.

\section{Working Memory}

As was mentioned previously, sensitivity was poorer in the color WM task than in the location WM task. More than half of all the participants reported using a verbal representation of the critical color, suggesting that the color task may have accessed verbal WM stores, which is consistent with Baddeley and Hitch's (1994) dissociation of verbal and spatial WM buffers. The overall differences in sensitivity to the two tasks may have arisen because there were two possible colors in which a wrench could appear but four possible locations in which the wrench head could appear. Thus, the color task may have been more susceptible to cumulative interference effects that arose as each item was repeated.

Task sensitivity did not change reliably over time for either WM task. Several factors may account for this stability. First, the participants were given several practice blocks, which may have reduced the likelihood of a sensitivity decrement (Fisk \& Schneider, 1981). Second, the match probability was $50 \%$; target probabilities typically must be low to produce a vigilance decrement (Davies \& Parasuraman, 1982). In addition, the wrench stimuli remained on the screen for 3,900 of the 4,000 msec of each trial, making the signal strength for each stimulus fairly high. The oval stimuli for the vigilance task, on the other hand, flashed briefly, reducing the signal strength and increasing the likelihood of a vigilance decrement (Teichner, 1974). Furthermore, although the particular orientation, color, and depth combination of wrench stimuli was unpredictable from trial to trial, the wrench pairs were always presented centrally. Therefore, spatial uncertainty, which can reduce vigilance performance (Warm, Dember, Murphy, \& Dittmar, 1992), may have played a less important role in performance of the WM task than in the vigilance task. Thus, although cognitive control was required to perform the
WM tasks throughout the test period, other aspects of the task were not ideal for producing a sensitivity decrement.

\section{The Relationship Between Working Memory and Vigilance}

The neural mechanisms mediating the link between WM and vigilance demonstrated in this study have not been systematically investigated. Evidence from lesion (e.g., Rueckert \& Grafman, 1998) and functional neuroimaging (e.g., Paus et al., 1997) studies indicates that some of the cortical brain regions subserving WM performance, such as the right prefrontal and posterior parietal cortex (Smith \& Jonides, 1998), may also mediate changes in detection performance over time. Cohen and ServanSchreiber (1992) also have provided evidence, using a connectionist model approach, that selective attention and WM deficits in schizophrenics result from an inability to form and maintain an internal representation of context for targets and nontargets. Although Cohen and ServanSchreiber modeled performance on the continuous performance test (CPT) to explain selective attention deficits, Nestor, Faux, McCarley, Shenton, and Sands (1990) found larger performance decrements over time on a CPTlike task for schizophrenics than for healthy controls, suggesting an important role for contextual maintenance in vigilance as well. Functional neuroimaging studies provide some evidence for the role of a contextual maintenance mechanism in vigilance decrement. Prefrontal cortical activity increases initially but declines over time during the performance of a vigilance task (Paus et al., 1997).

\section{CONCLUSIONS}

What do the results suggest about the relationship between WM and sustained attention? We propose that target discrimination tasks depend on WM to compare current test stimuli with recently encountered stimuli and with the template target item held in memory, particularly when perceptual comparison is difficult due to the successive presentation of stimuli or to the poor perceptual quality of the stimuli. In such cases, the storage and cognitive control processes of the WM system benefit from increased activation provided by subcortical arousal mechanisms. As the task is performed continuously, the resources available to the supporting vigilance systems diminish, as does the amount of computational support that these systems can provide. This decreased support can manifest in a poorer quality representation of items in memory or in the decreased efficiency of cognitive control processes, depending on task demands.

The goal of this study was to examine whether WM representation plays a specific role in vigilance decrement. The results provide strong evidence for such a role. However, one might predict, in addition, that if a verbal vigilance task had also been used, a greater sensitivity decrement would have occurred in the color memory dualtask condition than in the location memory condition. Although such a double dissociation would provide even 
more convincing evidence for the role of modality-specific memory representation in vigilance decrement, obtaining such evidence might raise problems. Verbal vigilance tasks in which alphanumeric stimuli are used often show no evidence of vigilance decrement, possibly due to the high familiarity of alphanumeric stimuli (See, Howe, Warm, \& Dember, 1995), making it difficult to test such a prediction. Nevertheless, this issue would be worth exploring in a future study, given that a verbal task sensitive to vigilance decrement could be developed. Furthermore, an anonymous reviewer observed that modality-specific processing demands, rather than memory storage demands, may have accounted for the increased vigilance decrement in the spatial WM condition. Previous research has demonstrated that an increase in nonmnemonic spatial processing demand (e.g., detecting an infrequent target that can occur in several possible locations in a visual field vs. just one location) does impair the overall level of vigilance performance. However, spatial processing demand does not increase the vigilance decrement (if anything, the decrement is left unchanged or reduced; Parasuraman, 1986), whereas WM demand does increase the decrement (Parasuraman, 1979). Hence, it is unlikely that the increased vigilance decrement in the location WM condition, as compared with the color WM condition, was due to spatial processing per se, rather than to the need for spatial WM. Nevertheless, it may be worthwhile to test the possibility in a future study by pairing nonmnemonic and mnemonic spatial tasks with a spatial vigilance task.

\section{REFERENCES}

Baddeley, A. [D.], Cocchini, G., Della Sala, S., Logie, R. H., \& SPINNLER, H. (1999). Working memory and vigilance: Evidence from normal aging and Alzheimer's disease. Brain \& Cognition, 41, 87-108.

Baddeley, A. D., \& Hitch, G. J. (1994). Developments in the concept of working memory. Neuropsychology, 8, 485-493.

Cohen, J. D., \& Servan-Schreiber, D. (1992). Context, cortex, and dopamine: A connectionist approach to behavior and biology in schizophrenia. Psychological Review, 99, 45-77.

Davies, D. R., \& Parasuraman, R. (1982). The psychology of vigilance. London: Academic Press.

Dobbs, A. R., \& Rule, B. G. (1989). Adult age differences in working memory. Psychology \& Aging, 4, 500-503.

FISK, A. D., \& SCHNEIDER, W. (1981). Control and automatic processing during tasks requiring sustained attention: A new approach to vigilance. Human Factors, 23, 737-750.

Gluckman, J. P., Dember, W. N., \& WArm, J. S. (1988). Capacity demand in dual task monitoring of simultaneous and successive vigilance tasks. Proceedings of the Human Factors Society, 32, 1463-1465.

Green, D. M., \& Swets, J. A. (1974). Signal detection theory and psychophysics. Huntington, NY: Krieger.

JAMES, W. (1890). Principles of psychology. New York: Holt.

Kramer, A. F., \& Weber, T. A. (1998). Object-based attentional selection and aging. Psychology \& Aging, 14, 99-107.

MACKWORTH, J. F. (1969). Vigilance and habituation. Baltimore: Penguin.

Nestor, P. G., Faux, S. F., McCarley, R. W., Shenton, M. E., \& Sands, S. F. (1990). Measurement of visual sustained attention in schizophrenia using signal detection analysis and a newly developed computerized CPT task. Schizophrenia Research, 3, 329-332.

Nuechterlein, K. H., Parasuraman, R., \& Jiang, Q. (1983). Visual sustained attention: Image degradation produces rapid sensitivity decrement over time. Science, 220, 327-329.
Parasuraman, R. (1979). Memory load and event rate control sensitivity decrements in sustained attention. Science, 205, 924-927.

Parasuraman, R. (1986). Vigilance, monitoring, and search. In K. R. Boff, L. Kaufman, \& J. P. Thomas (Eds.), Handbook of perception (pp. 43.1-43.49). New York: Wiley.

Parasuraman, R., \& Mouloua, M. (1987). Interaction of signal discriminability and task type in vigilance decrement. Perception \& Psychophysics, 41, 17-22.

Paus, T., Zatorre, R. J., Hofle, N., Caramanos, Z., Gotman, J., Petrides, M., \& Evans, A. C. (1997). Time-related changes in neural systems underlying attention and arousal during the performance of an auditory vigilance task. Journal of Cognitive Neuroscience, 9, 392408.

Rueckert, L., \& Grafman, J. (1998). Sustained attention deficits in patients with lesions of posterior cortex. Neuropsychologia, 36, 653660.

See, J. E., Howe, S. R., Warm, J. S., \& Dember, W. N. (1995). Metaanalysis of the sensitivity decrement in vigilance. Psychological Bulletin, 117, 230-249.

SMITH, E. E., \& JONIDES, J. (1998). Neuroimaging analyses of human working memory. Proceedings of the National Academy of Sciences, 95, 12061-12068.

TeICHNER, W. H. (1974). The detection of a simple visual signal as a function of time of watch. Human Factors, 16, 339-353.

Todkill, A. L., \& HuMPhreYS, M. S. (1994). Stimulus comparison strategies and task demands in successive discrimination. Quarterly Journal of Experimental Psychology, 47A, 761-779.

WARM, J. S., \& DEMBER, W. N. (1998). Tests of vigilance taxonomy. In R. R. Hoffman, M. R. Sherrick, \& J. S. Warm (Eds.), Viewing psychology as a whole (pp. 87-112). Washington, DC: American Psychological Association.

Warm, J. S., Dember, W. N., Murphy, A. Z., \& Dittmar, M. L. (1992). Sensing and decision-making components of the signal-regularity effect in vigilance performance. Bulletin of the Psychonomic Society, 30, 297-300.

WiCKENS, C. D. (1984). Processing resources in attention. In R. Parasuraman \& D. R. Davies (Eds.), Varieties of attention (pp. 63-102). San Diego: Academic Press.

\section{NOTES}

1. In a posttask questionnaire, we asked participants to state whatever type of strategy they may have used to perform the task. We intentionally did not ask specifically about verbal or spatial strategies to avoid experimental demand effects on what strategy the participants adopted during the subsequent test condition. Strategies were classified as verbal if they indicated that the participants had used subvocal rehearsal (e.g., "thinking what color the ' $\mathrm{C}$ ' had to be on next by saying it in my head") and spatial if they indicated either a visualization (e.g., "I visualized how the wrench needed to rotate in order to match the one 2 previously. ..") or an eye movement strategy (e.g., "My strategy was to look at where the 'C' would have to be in order for it to be a match . ..'). All participant responses that did not indicate a clearly defined use of either a verbal or a spatial strategy involved either a nonspecific restatement of the task instructions (e.g., "I compared the color to the color from the trial two previously") or an attempt to divine a pattern in the order in which match and nonmatch trials were presented. Thus, although many participants did not report using the strategy we had anticipated, the way in which the question was worded suggests that they may, in fact, have used such a strategy but failed to report it. Although this method of questioning was susceptible to nondescript answers that provided no insight into the specific issue we wanted to address, we deemed it necessary to avoid implicitly prompting the participants to attempt verbal strategies for the location WM task when it was performed as the second condition by explicitly inquiring about verbal and spatial strategies.

2 . We are grateful to an anonymous reviewer for this suggestion.

(Manuscript received July 17, 2003; revision accepted for publication November 14, 2003.) 